\begin{tabular}{|c|c|}
\hline \multirow{3}{*}{ 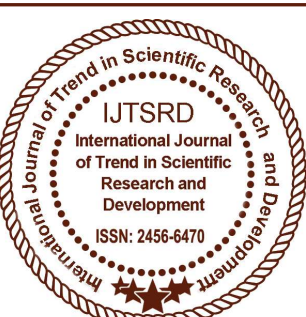 } & $\begin{array}{l}\text { International Journal of Trend in Scientific } \\
\text { Research and Development (IJTSRD) }\end{array}$ \\
\hline & national Open Access Journal \\
\hline & ISSN No: $2456-6470$ | www.ijtsrd.com | Volume - 2 | Issue - 5 \\
\hline
\end{tabular}

\title{
Image Compression Using Fractal Dimension Based on Quadtree Fuzzy Logic Method
}

\author{
Rachna Srivastava ${ }^{1}$, Yogesh $\mathrm{Pal}^{2}$ \\ ${ }^{1}$ Research Scholar, ${ }^{2}$ Assistant Professor \\ Department CSE, BIET, Lucknow, Uttar Pradesh, India
}

\begin{abstract}
We have made image/photo compression the use of efficient fuzzy good judgment on this research. We have used quadtree set of rules for this motive. We choose fuzzy common sense primarily based method as fuzzy good judgment is taken into consideration strong tool to deal with vagueness. When photos are indistinct in phrases of pixel values fuzzy common sense is taken into consideration appropriate common sense for its analysis. In proposed technique one domain block is taken into consideration for every range block \& searched best for matched comparison scaling. So effects fractal code does now not contain coordinates of matched area block. Quadtree set of rules can be here applied in such case \& length of range block may be minimized as small as $2 \times 2$ pixels. Proposed research offers with integration of quad tree algorithm with traditional DCT primarily based fractal photo compression as a way to produce higher compression ratio PSNR with less compression error. Our fundamental goal is to review the photograph of excessive compression and backbone and which will compress it we use a novice algorithm and implements it in photograph so that the pixels of the photo got compressed. In order to reap this objective we use Fuzzy Logic.
\end{abstract}

Keywords: DCT, Image Processing, Fuzzy Logic, PSNR, and Quad tree Algorithm

\section{INTRODUCTION}

Every single day world is evolving very rapid. Rapid development of the technology has affected all of the clinical areas. Medicine, automation, facts analysis, finances, biology, chemistry, economics and lots of, many extra have benefited from the generation

growth. Those huge modifications have additionally inspired fields as an photo processing.

Image enhancement tactics include a set of strategies that are seeking for to improve the visual appearance of an photograph or to convert the photo to a form better perfect for analysis through a human or a device. Enhancement of noisy photo records is a totally hard issue in lots of research and application areas. Image enhancement strategies can be divided into three wide categories:

1. Spatial area strategies, which perform at once on pixels using gray degree variations or histogram processing (classical histogram equalization).

2. Frequency area strategies, which operate at the Fourier remodel of an photo.

3. Fuzzy domain techniques, which contain the usage of expertise-base structures that are able to mimicking the behavior of a human expert the usage of fuzzy based histogram equalization.

Classical Histogram Equalization (HE) has proved to be a easy and effective picture assessment enhancement technique .But this has a drawback that it does now not hold the brightness of the enter photo at the output one. This makes HE not suitable for photo comparison enhancement on purchaser electronic products, including video surveillance, in which maintaining the enter brightness is critical to avoid the era of non-existing artifacts inside the output picture. To triumph over such downside, versions of the classic $\mathrm{HE}$ technique have proposed to first decompose the enter image into two sub-pictures, after which carry out HE independently in every sub- 
photograph. Although those techniques preserve the enter brightness at the output picture with a widespread evaluation enhancement, they'll produce pics which do no longer appearance as herbal as the enter ones.

Fuzzy logic represents an excellent mathematical framework to cope with uncertainty of information. Fuzzy photograph processing is the collection of all methods that understand constitute and system the snap shots, their segments and features as fuzzy sets.

In the present thesis, an set of rules is proposed for Fuzzy Histogram Equalization. This set of rules complements picture comparison as well as preserves the brightness very efficaciously. This additionally reduces its computational complexity. This Fuzzy Histogram Equalization method uses the representation and processing of digital image in fuzzy information. These pics in fuzzy area deal with the inexactness of gray-stage values in a better manner compared to GHE and CLAHE like traditional techniques, which improves its overall performance. Hence, proposed Fuzzy set of rules can be used for picture enhancement of poor satisfactory photos. All the implementation paintings has been carried out in MATLAB 7.5 Image Processing device field.

Data representation in Matlab is the function that distinguishes this surroundings from others. Every statistics is presented with matrices. The definition of matrix is a rectangular array of numbers. Most photographs are kept in -dimensional matrices. Each element corresponds to 1 pixel in the picture. True shade snap shots require a 3rd size to hold the statistics approximately intensities of RGB colorings. Fuzzy Logic Toolbox gives wide range of functions liable for fuzzy calculations. It permits consumer to glance through the outcomes of fuzzy computations. Experimental consequences display that the satisfactory of photo is improved after fuzzy histogram equalization. It is examined on one of a kind commonplace formats of photographs taking distinctive fuzzy membership characteristic.

Our main objective is to review the photo of excessive compression and resolution and if you want to compress it we use a beginner set of rules and implements it in photo so that the pixels of the photograph were given compressed.
In order to acquire this goal we use Fuzzy Logic. Fuzzification of an photograph leads to the reduction inside the evaluation and brightness of input photograph to be compressed. The advantage of this discount in evaluation and brightness is this discount ends in growth the pixel redundancy and as a result assist to growth the compression ratio (CR) and height sign to noise ratio (PSNR) all through the photograph compression. And the algorithm which we are using is Quad tree Algorithm. Through Quad tree Algorithm the scale of the range block can be lessen as small as $2 X 2$ pixels. Hence the great of decoded picture can be improved while the compression ratio can be maintained.

\section{RELATED WORK}

In 2006 Yu-fai Fung, Homan Lee, and M. FikretErcan worked on software of toll rate charged for the usage of facilities which includes a tunnel or a bridge is normally proportional to the wide variety of axles possessed by using a automobile. They designed an automatic system that can perceive the range of axles is sought. Instead of detecting the axle, wheels of a car were examined and a technique based totally at the Hough transform for detecting circles turned into proposed. As the machine have to be capable of locate the suitable quantity of wheels in real-time, subsampling based totally on the Haar Wavelet transform changed into carried out. The method turned into capable of pick out the wheel effectively to procedure the enter photographs in actual-time.

They conclude that the Hough rework is suitable for such an utility. It can procedure as much as 24 photos within 1.Five $\mathrm{s}$ and it happy the timing constraint imposed upon the system. The gadget setup turned into easy and through using commodity components, its setup fee changed into additionally low.

In 2006 ZhiYu Chen, Besma R. Abidi, David L. Page, Mongi A. Abidi gave a contrast enhancement has an crucial role in photograph processing packages. They defined that conventional contrast enhancement strategies both often fail to produce first-class consequences for a large variety of low-evaluation pictures, or can't be robotically applied to one of a kind image, due to the fact their parameters need to be distinct manually to provide a excellent result for a given photo. They described a brand new automated approach for contrast enhancement. First of all they grouped the histogram components of a lowcomparison photo right into a right wide variety of 
bins in line with a selected criterion, then redistributed those packing containers uniformly over the grayscale, and in the end ungroup the previously grouped gray-tiers. Accordingly, this new method is called grey-stage grouping (GLG). GLG now not only produces outcomes superior to traditional assessment enhancement techniques, however is likewise completely computerized in most situations, and is applicable to a large kind of images. An extension of GLG is selective GLG (SGLG). SGLG selectively businesses and ungroups histogram components to obtain particular utility functions, which includes doing away with background noise, improving a selected section of the histogram, and so forth.

They evolved a new automatic assessment enhancement approach. GLG became a trendy and effective technique, which can be effectively implemented to a large sort of low-comparison pictures and generates first-class outcomes. HE method will be carried out with full automation at fast speeds.

In 2007 David Menotti, Laurent Najman, Jacques Facon, and Arnaldo de A. Araújo proposed that Histogram equalization (HE) has proved to be a easy and effective image comparison enhancement technique. They labored on a novel method called Multi-HE, which includes decomposing the enter picture into several sub-pics, and then applying the classical HE procedure to every one. This method plays a less extensive image assessment enhancement, in a way that the output picture affords a extra herbal look. They proposed discrepancy capabilities for picture decomposing, conceiving two new Multi-HE strategies. A value function become extensively utilized for routinely finding out in how many subpics the enter image could be decomposed on. The work become tested a brand new framework called MHE for picture evaluation enhancement and brightness maintaining which generated herbal searching pictures. The results showed that there methods changed into higher on keeping the brightness of the processed picture (in relation to the original one) and yields pics with herbal look, on the value of assessment enhancement.

In 2009 Tarik Arici, SalihDikbas, and YucelAltunbasak gave a standard framework based totally on histogram equalization for photo contrast enhancement is offered. In this framework, comparison enhancement is posed as an optimization problem that minimizes a cost feature. They added especially designed penalty terms, the level of evaluation enhancement may be adjusted; noise robustness, white/black stretching and meanbrightness preservation might also easily be included into the optimization. Analytic answers for a number of the critical criteria had been provided. Finally, a low-complexity set of rules for assessment enhancement changed into presented, and its performance was validated towards a recently proposed technique.

\section{METHODOLOGY}

In imaging science, image processing is any form of signal processing for which the enter is an photograph, inclusive of a image or video body; the output of photograph processing may be either an photograph or a hard and fast of characteristics or parameters related to the photograph. Most photograph-processing techniques contain treating the photo as a two-dimensional sign and making use of wellknown sign-processing techniques to it.

Image processing typically refers to virtual photo processing, but optical and analog photograph processing are also feasible. The acquisition of snap shots (producing the enter photograph in the first location) is known as imaging. An image can be considered to incorporate sub-pics once in a while known as regions-of-interest, ROIs, or virtually regions. This idea reflects the fact that pix often incorporate collections of objects every of which may be the basis for a place. In a sophisticated image processing gadget it ought to be feasible to apply particular picture processing operations to selected areas. Thus one a part of an picture (place) might be processed to suppress motion blur even as every other element is probably processed to improve colour rendition.

Image processing is a method to convert an image into virtual shape and carry out a few operations on it, with a purpose to get an more suitable photograph or to extract a few beneficial records from it. It is amongst hastily developing technology these days, with its applications in various elements of a enterprise. Image Processing forms core studies region inside engineering and computer science disciplines too.

Image processing essentially includes the following 3 steps: 
1. Importing the picture with optical scanner or with the aid of virtual photography.

2. Analyzing and manipulating the photograph which incorporates information compression and photograph enhancement and spotting patterns that are not to human eyes like satellite tv for pc snap shots.
3. Output is the remaining degree in which end result can be altered picture or document that is primarily based on picture analysis.

Fundamental steps in virtual picture processingare

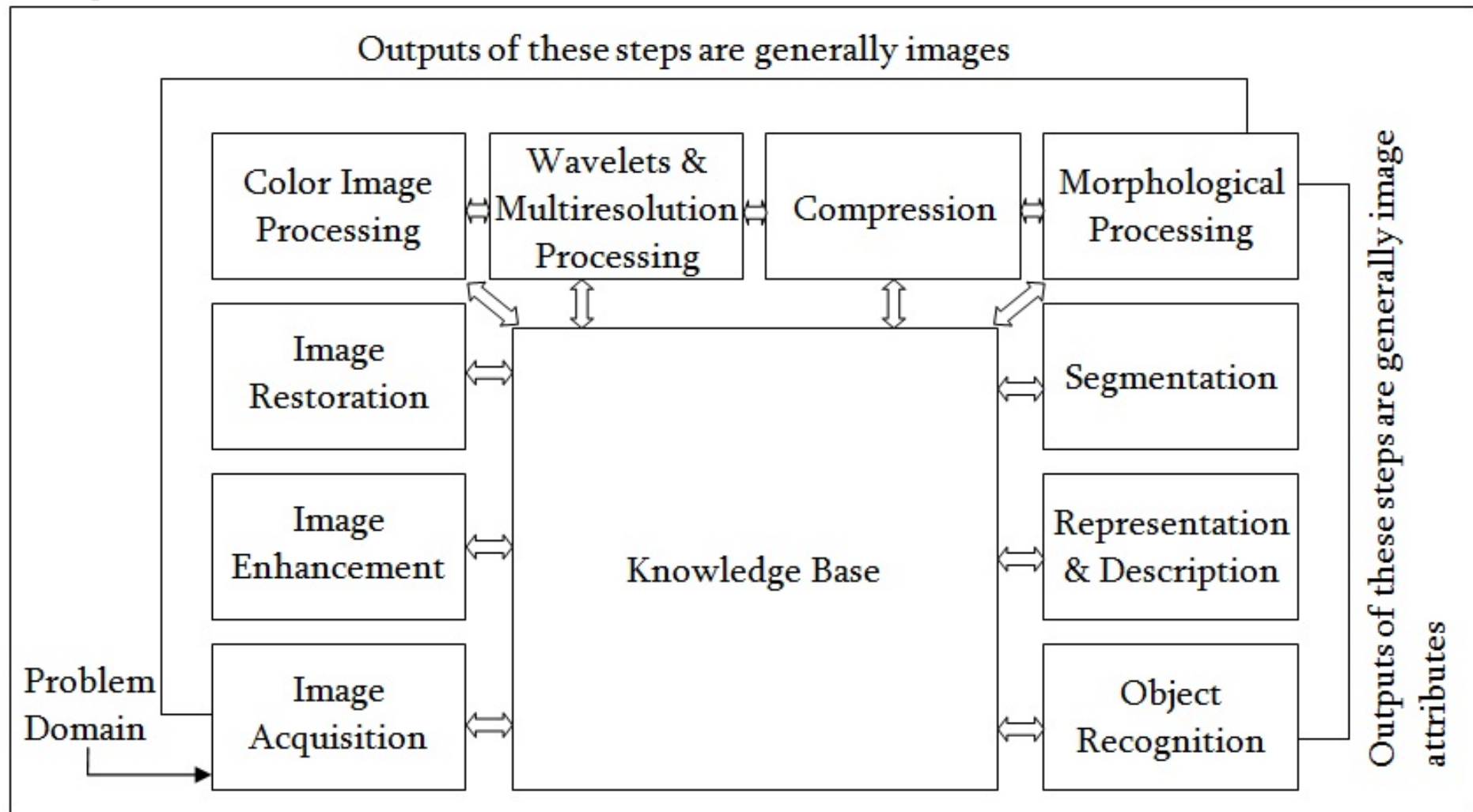

\section{ALGORITHM:}

$>$ In this implementation first of all the algorithm reads an image and defines the size of the range blocks \& domain blocks.

$>$ As per the defined size of range blocks and domain blocks the algorithm breaks the image in respective horizontal and vertical address of blocks.

> The image blocks of size 16 X 16 are saved as TP. These blocks are further modified by reducing the pixel values by half.

$>$ DCT is applied on each block and saved as TRR. Same operation is to be performed on domain blocks and they are saved as TD in a size of $32 \mathrm{X}$ 32 .

$>$ Then the DCT of domain blocks are saved as TDM.

Then the domain blocks are down sampled to the size of $16 \times 16$. Then the error between range blocks and domain blocks will be evaluated.

$>$ As per the array values fuzzy logic will decide the no. of fractals prior to applying encoding the pixels.

\section{RESULT AND DISCUSSION}

The results of fractal image compression using fuzzy logic decision mechanism is explained in detailed manner. The algorithm is developed on MATLAB based platform using image processing and fuzzy logic toolbox. Several cases are considered for standard gray scale image to generate compressed image and then the compressed image is decompressed back to visualize the original and recovered image under different test cases. The losses due to approximation of domain block and range block differences makes failure of high quality of recovery of image data. Due to this errors are found in between recovered and original image for analyzing the result performance the PSNR values are measured for different error threshold condition.

The level of compression is most important factor and it is determined by evaluation the compression ratio i.e. ratio of the size of original image upon the size of compressed image. PSNR is evaluated in between original image and decompressed image. The 
compression ratio should be as high as possible but not at the cost of PSNR. Image recovery is assumed to be satisfactory if the PSNR is found to be above than 30 .

We have considered two standard images lena.jpg and jet, jpg for test the algorithm performance and evaluated the compression ration and PSNR using original image, compressed image and decompressed (recovered image).The fuzzy logic rule decides for further fractal decomposition of image 16x16 blocks if the difference between range and domain block is larger than a threshold. If after the fractal decomposition into quadraturethe error in between new and range and domain blocks is higher than next level threshold then again $8 \times 8$ block is further passed through the fractal decomposition. The error thresholds are named as Err1,Err2 and Err3 such that Err $>$ Err2 $>$ Err3.

The fuzzy rules are designed as:

If err between 16x16 size range block and domain block $<$ Err1

Then perform encoding of difference of range block and domain block

Else perform fractal decomposition and generate quadrature range blocks of size $8 \times 8$
The error threshold we have taken at different combinations and performed image compression and decompression of jet.jpg and lena.jpg image and find the PSNR and CR values to decide the optimum threshold that can be considered to generate the best compression quality.

The different thresholds are considered in decreasing order and given below:

\section{Table 1: Errors threshold values for different image compression analysis test cases.}

\begin{tabular}{|l|l|l|l|}
\hline $\begin{array}{c}\text { S. } \\
\text { No. }\end{array}$ & $\begin{array}{c}\text { Errors } \\
\text { Threshold } \\
\text { E1 }\end{array}$ & $\begin{array}{c}\text { Errors } \\
\text { Threshold } \\
\text { E2 }\end{array}$ & $\begin{array}{c}\text { Errors } \\
\text { Threshold } \\
\text { E3 }\end{array}$ \\
\hline 1 & 10 & 8 & 6 \\
\hline 2 & 10 & 6 & 2 \\
\hline 3 & 10 & 5 & 1 \\
\hline 4 & 8 & 7 & 6 \\
\hline 5 & 8 & 6 & 4 \\
\hline 6 & 8 & 4 & 1 \\
\hline 7 & 6 & 4 & 3 \\
\hline 8 & 6 & 3 & 1 \\
\hline $90 u$ & 6 & 2 & 0.5 \\
\hline 10 & 4 & 3 & 2 \\
\hline 11 & 4 & 2 & 1 \\
\hline 12 & 4 & 1 & 0.5 \\
\hline 13 & 3 & 2 & 1 \\
\hline 14 & 2 & 1 & 0.5 \\
\hline
\end{tabular}

If err between $8 \times 8$ size range block and domain block $<$ Err2

Then perform encoding of difference of range block and domain block

Else perform fractal decomposition and generate quadrature range blocks of size $4 \times 4$

If err between $4 \times 4$ size range block and domain block $<$ Err3

Then perform encoding of difference of range block and domain block

Else perform fractal decomposition and generate quadrature range blocks of size $2 \times 2$ and perform encoding of difference of range block and domain block of size $2 \times 2$.

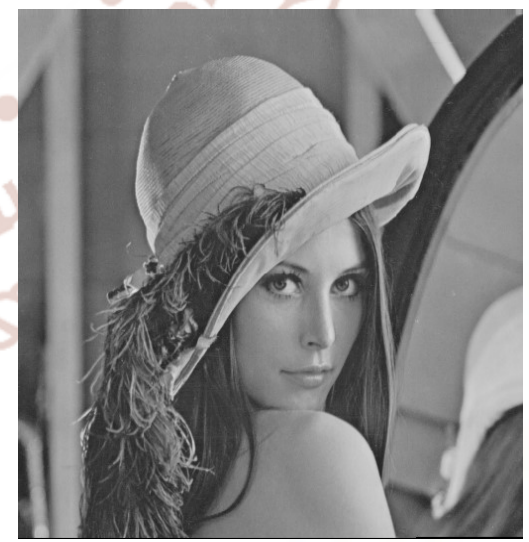

Fig.2. 'leena.jpg'original image

Figure 2 shows the original image of leena.jpg on passing this image by fuzzy based fractal wavelet image compression the decompressed images are shown in figure 2. 


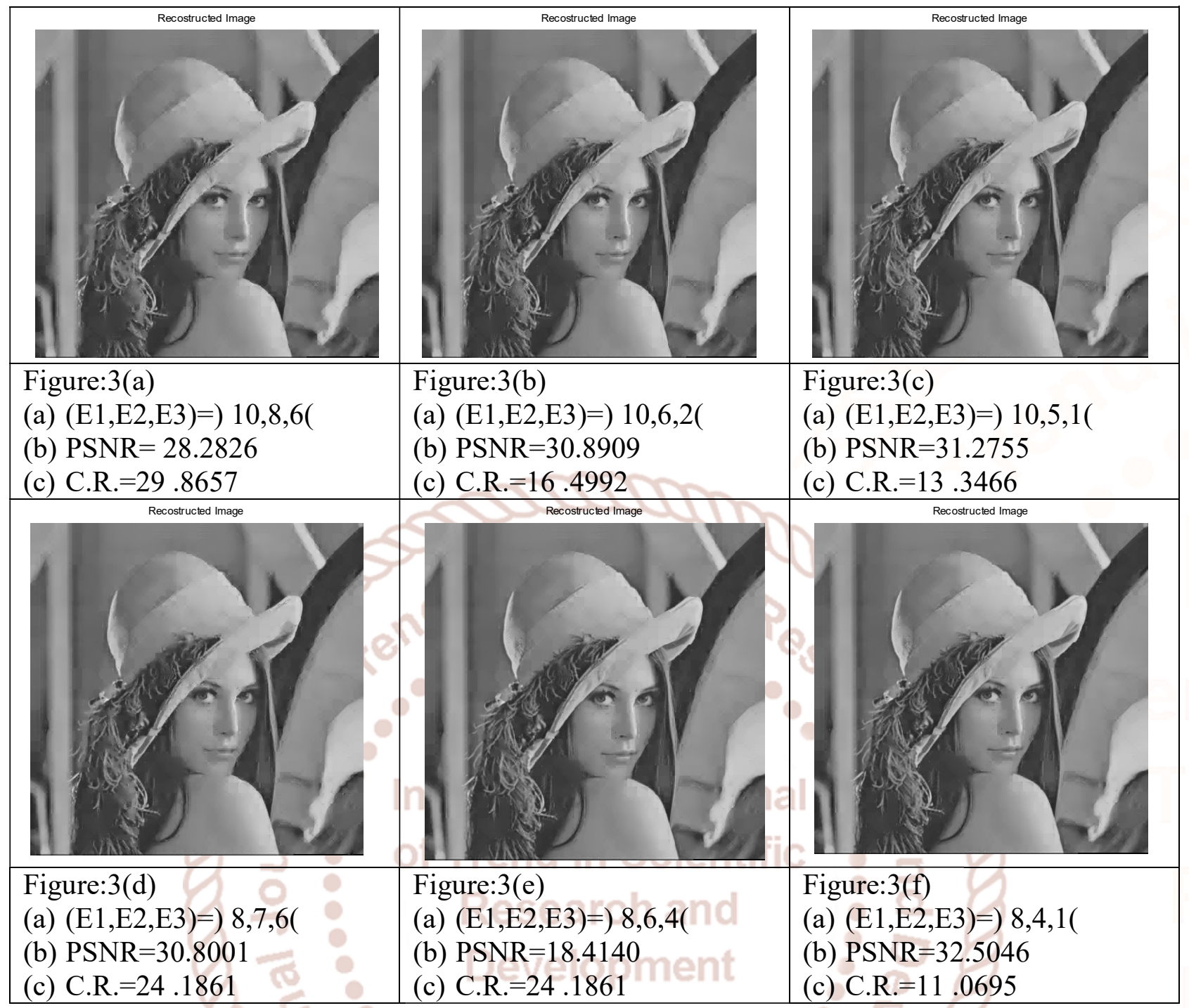

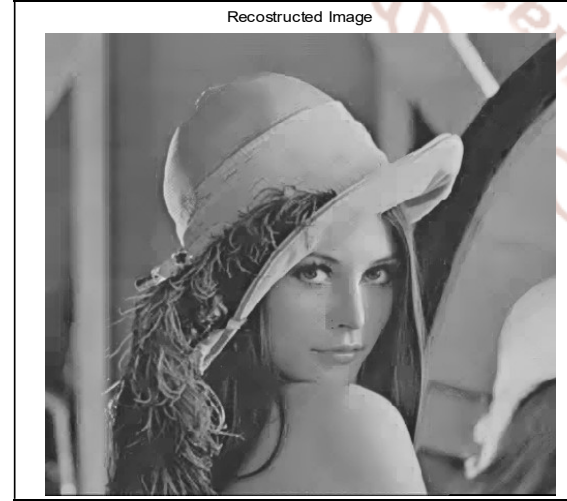

Figure:3(g)
(a) $(\mathrm{E} 1, \mathrm{E} 2, \mathrm{E} 3)=) 6,4,3($
(b) $\mathrm{PSNR}=33.1988$
(c) C.R. $=13.3692$

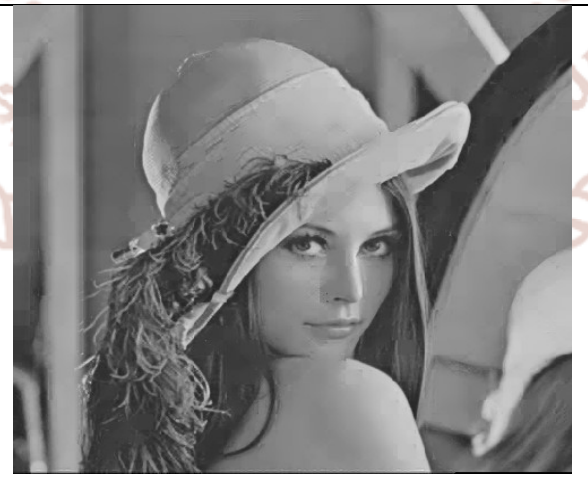

Figure:3(h)
(a) $(\mathrm{E} 1, \mathrm{E} 2, \mathrm{E} 3)=) 6,3,1($
(b) $\mathrm{PSNR}=33.8508$
(c) C.R. $=8.7230$

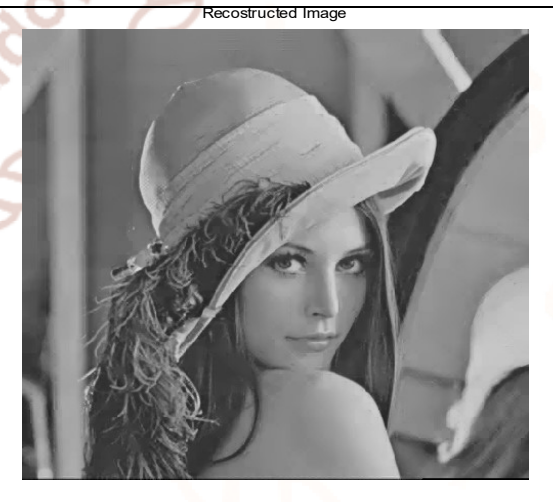

Figure:3(i)

(a) $(\mathrm{E} 1, \mathrm{E} 2, \mathrm{E} 3)=) 6,2,0.5($

(b) $\mathrm{PSNR}=34.2213$

(c) C.R. $=6.8971$ 


\begin{tabular}{|l|l|}
\hline &
\end{tabular}

Figure 3 (a to 1) the decompressed images of lena.jpg at different error thresholds are shown the value of PSNR and CR for these results are plotted below:

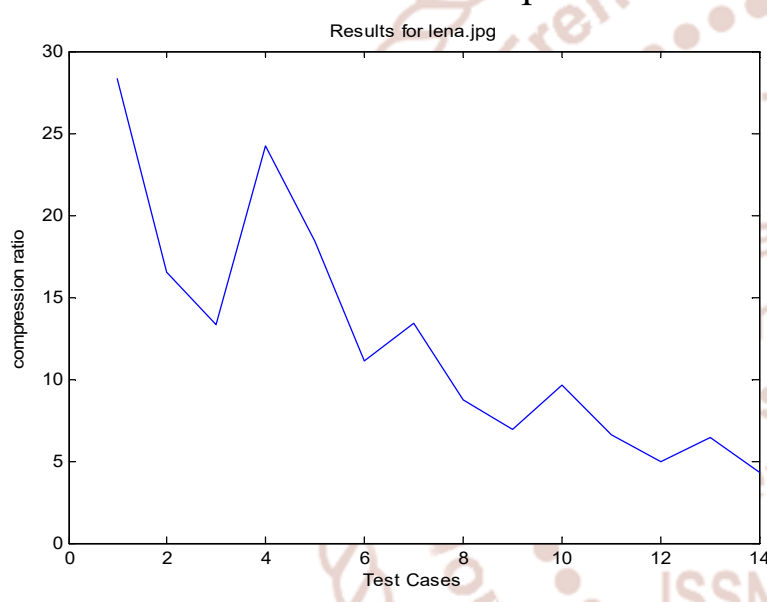

Fig 4: Compression Ratio for lena.jpg image at different error threshold cases.

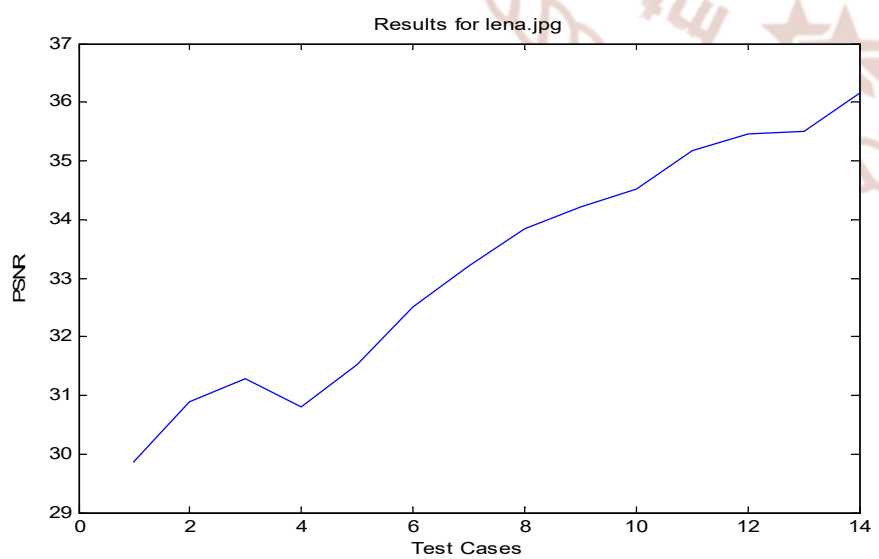

Fig 5: PSNR for lena.jpg image at different error threshold cases.

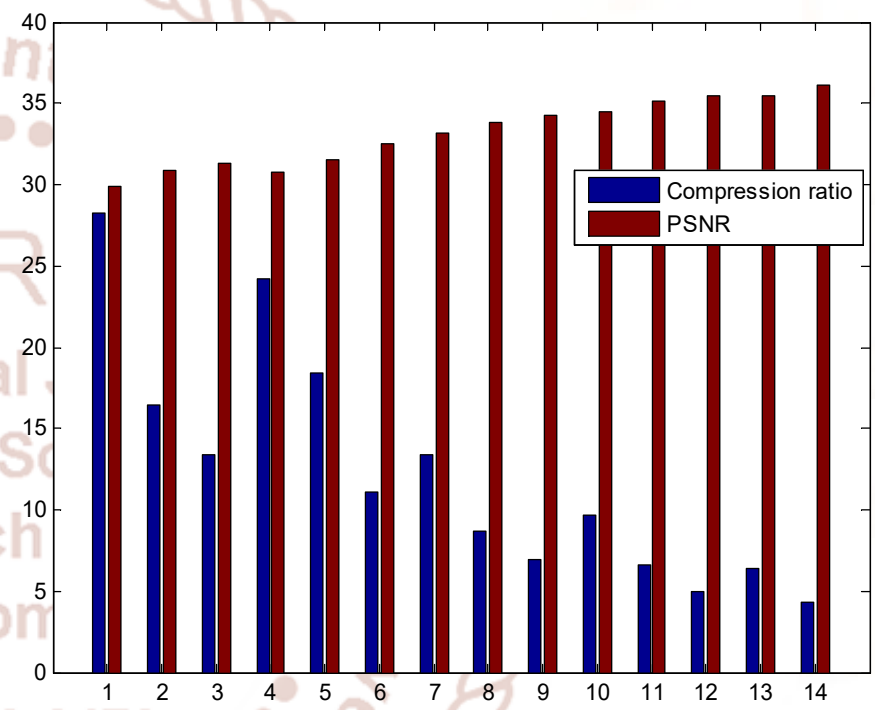

Fig 6: Compression Ratio and PSNR for lena.jpg image at different error threshold cases.

Table 2: PSNR Compression [11]

\begin{tabular}{|l|l|l|}
\hline & $\begin{array}{l}\text { Previous Work } \\
{[11]}\end{array}$ & Our Work \\
\hline PSNR & 27.45 & 31.2755 \\
\hline CR & 12.8 & 13.3466 \\
\hline
\end{tabular}

\section{CONCLUSION:}

In this painting a excessive diploma of photo compression algorithm is developed and examined on digital photo. For this motive literature survey of various traditional picture compression schemes used to encode the pix with the aid of pixel-primarily based and statistical techniques is achieved. Fractal based totally picture compression is observed to be exciting and efficient at the shape-based photograph compression techniques. Fractal photograph compression is implemented and is located that it is able to be used no longer best in photo compression 
coding however also in different photo processing packages like sample popularity and biometric identity. The most important drawback on this algorithm became that this photograph coding generation of compression faces high computational complexity in encoding because of involvement of image partitioning into some rectangular fractals and then those rectangular fractals compose a massive pool set. Because of different form of block length an photograph is partitioned into extraordinary pool units. These pool units composed of the blocks of huge length is known as the area pool set and the other pool set is called because the range pool set. The cells of the variety pool set blocks are encoded. The blocks inside the area pool set are gotten smaller in the identical size as the range blocks pool set, and then fractal photograph compression takes the domain pool set fee as a virtual list of the codebook. In this work we have applied no searching of minimum distinction matching area block from domain pool set for each range block which changed into to be searched in preceding algorithms. In this manner this set of rules of fractal image compression eliminated the exhaustive search of required in matching block pair's prices and time, that's one of the primary problems in Fractal photograph compression. On destiny we can involve other A.I. Techniques like neural community, ANFIS in hybrid with fuzzy good judgment for finding out mechanism of photograph sub fractals.

\section{REFERENCES:}

1. Gonzalez, R. and Woods, R., 2002. Digital Image Processing, Prentice Hall.

2. Wang, C. and Ye, Z., 2005. Brightness Preserving Histogram Equalization with Maximum Entropy: A Variational Perspective. IEEE Trans. Consumer Electronics, Vol. 51, Issue 4, pp. 1326-1334.

3. Menotti, D., Najman, L., Facon, J., Araújo, A.A., 2007. Multi-Histogram Equalization Methods for
Contrast Enhancement and Brightness Preserving. IEEE Trans. on Consumer Electronics, Vol. 53, Issue 3, pp.1186-1194.

4. Cheng, H.D., Shi, X.J., 2004. A simple and effective histogram equalization approach to image enhancement. Digital Signal Processing, Vol.14, pp. 158-170.

5. Chen, Z., Abidi, R., Page, D.L., Abidi, A., 2006. Gray-Level Grouping (GLG): An Automatic Method for Optimized Image Contrast Enhancement-Part I: The Basic Method. IEEE Trans. On Image Processing, Vol. 15, Issue 8, pp. 2290-2302.

6. Chen, S.D. and Ramli, A.R., 2004. Preserving brightness in histogram equalization based contrast enhancement techniques. Digital Signal Processing, Vol.14, Issue 5, pp. 413-428.

7. Yoon, H., Han, Y., Hahn, H., 2009. Image Contrast Enhancement based Sub-histogram Equalization Technique without Over-equalization Noise. World Academy of Science, Engineering and Technology, Vol. 26, pp. 176-182.

8. Arici, T. and Dikbas, S., 2009. A Histogram Modification Framework and Its Application for Image ContrastEnhancement. IEEE Trans. On Image Processing, Vol. 18, Issue 9, pp. 19211935.

9. Chanda, B. and Majumder, D.D., 2002. Digital Image Processing and Analysis, Prentice Hall.

10. Kim, Y.T., 1997. Contrast Enhancement Using Brightness Preserving Bi-Histogram Equalization. IEEE Trans. Consumer Electronics, Vol. 43, Issue 1 , pp. 1-8

11. Shuai Liuet.al., "A fractal image encoding method based on statistical loss used in agricultural image compression" Springer Science, Business Media New York 2015 